\title{
Development and Initial Validation of a Scale for Assessing Affecting Factors on Preventive Behaviors of COVID-19 (AFPB-CO): Using the Protection Motivation Theory
}

Maryam Khazaee-Pool ( $\square$ khazaie_m@yahoo.com ) Mazandaran University of Medical Sciences https://orcid.org/0000-0002-2587-3460

Seyed Abolhassan Naghibi ( $\sim$ anaghibi1345@yahoo.com )

Mazandaran University of Medical Sciences

Tahereh Pashaei

Kurdistan University of Medical Sciences

Leila Jahangiry

Tabriz University of Medical Sciences

Mahbobeh Daneshnia

Mazandaran University of Medical Sciences

Koen Ponnet

Universiteit Gent

Research article

Keywords: Scale development, Protection Motivation Theory, Preventive behaviors, COVID-19

Posted Date: April 27th, 2020

DOI: https://doi.org/10.21203/rs.3.rs-23051/v1

License: (c) (i) This work is licensed under a Creative Commons Attribution 4.0 International License.

Read Full License 


\section{Abstract \\ Background}

The protection motivation theory (PMT) is a practical theory for defining how people are interested in responding in a self-protective way concerning a perceived health threat. At present, there is no comprehensive, validated scale for measuring factors affecting on people' participation on preventive behaviors about COVID-19 in the world. A current study accomplished to develop and evaluate the validity and reliability of the theory-based scale using the protection motivation theory (PMT) constructs as a theoretical structure for measuring factors affecting on preventive behaviors of COVID-19.

\section{Design and methods:}

A multi-phase scale developmental method was applied to develop a scale for assessing affecting factors on preventive behaviors about COVID-19 (AFPB-CO) in 2020. The items for the AFPB-CO scale were made using three methods: literature review, interview with laypeople, and expert panel. To prepare a draft form of the AFPB-CO scale, we accomplished content and face validity analyses. The AFPB-CO scale validation was accompanied by a sample of laypeople, recruited from cyberspace and social networks. Finally, the construct validity of the AFPB-CO scale was assessed by applying both exploratory factor analysis (EFA) and confirmatory factor analysis (CFA). Internal consistency and test-retest methods were used for assessing the reliability of the AFPB-CO scale. Data were analyzed using SPSS and AMOS.

\section{Results}

Results obtained from factor analysis showed that the data were fit to the model $(\chi 2=5276.468, P<$ 0.001), and MPT consisted of 36 items assessing five domains, which describing $58.64 \%$ of the common variance. The CFA showed a model with suitable fitness for the data. Cronbach's alpha coefficient for the domains of the scale ranged from .62 to .84 , and the ICC value ranged from .73 to .87 , which is within satisfactory ranges.

\section{Conclusions}

The results from this study show that the Iranian version of the AFPB-CO has excellent psychometric properties and is appropriate for assessing the affecting factors on preventive behaviors among Iranian people toward COVID-19.

\section{Background}


Coronavirus disease 2019 (COVID-19) is an infectious disease that was first recognized in 2019 in Wuhan, China. Although, since it has feast worldwide, causing in the 2019-2020 coronavirus pandemic $(1,2)$. Having cough, fever, and breath tininess are common symptoms of COVID-19. It was also reported that having muscle pain, having a sore throat, losing smell or taste, producing sputum, having diarrhea, and abdominal pain may be other symptoms of COVID-19 $(3,4)$. The World Health Organization (WHO) confirmed the outbreak a Public Health Emergency of International Concern (PHEIC) on 30 Jan 2020 (8). The virus is mostly extent throughout near contact and through breathing drops created when individuals cough or sneeze (4-6). Time from contact to start of signs is commonly between 2-14 days. Furthermore, the infection can be detected from a grouping of risk factors, symptoms, and a chest CT scan displaying pneumonia features $(7,8)$.

Preventive methods to decrease the risks of infection contain remaining at home, being no in crowded places, cleaning hands with soap and warm water often and for as a minimum 20 seconds, touching no the mouth, eyes, or nose with dirty hands, and having social distancing about 1.80 meters (9). There is no vaccine or a distinct treatment for COVID-19 (7). It looks that the high rate of COVID-19 in the world is a complex event. Thus, educational interventions to reduce the rate of COVID-19 and improved the participation of people in preventive behaviors is the most potentially useful method to protect against COVID-19. Therefore, an instrument for assessing the factors affecting on people' participation in performing preventive behaviors about COVID-19 required.

One of the approaches to scale design, especially in the area of preventive behaviors, is the use of motivational theories like the protection motivation theory (PMT). PMT can be performed to describe behavior associated with threat and coping related to health dangers and behavioral intention. Concepts such as perceived severity, perceived vulnerability, perceived response cost, perceived response efficiency, and perceived self-efficacy are the key constructs of the protection motivation theory (PMT). These components are one of the most common reasons to do the prevention of disease people might consider themselves at risk of possible comorbidities. Threat references to the extent to which persons perceive they are vulnerable to the health danger and their opinion of the sternness of the health danger. Coping mentions to the extent individuals sense that a specific behavior will prevent them from the health danger and whether or not they sense they can accomplish such behavior. Self-efficacy had the most constant relation to intention, such that people were most probably to take action if they believed that they could successfully do an adaptive behavior. The PMT is suitable for realizing how beliefs participate in intention and behavior. The PMT may be applied to advise effective communications to reform existing postoperative recommendations (10). Thus, according to the reports mentioned above, PMT can be a suitable theory to design interventional programs.

Due to the importance of the participation in leading behavior, identifying underlying factors of preventive behavior are essential to improve any health promotion plan. As such, a valid and reliable questionnaire is required to extract affecting factors on participation in preventive behaviors. Based on the PMT, many instruments have been developed to measure to what extent interventions can cause improvement in persons' behavior. However, given that COVID-19 is the new disease of the present Century that has 
recently become Pandemic, so far, the researchers have not found any study and scale based on recent PMT in published documents. Thus, the current study was aimed to development and psychometric assessment of a multi-component questionnaire based on PMT better to understand factors affecting on preventive behaviors about COVID-19. Such scales could support to identify the viewpoints of health experts and policymakers and, in turn, help in developing extended interventional plans for participating of citizens about the rate of the preventive behaviors toward COVID-19.

\section{Methods}

\section{Study design}

The present work was applied in two separate stages. At the start, in the first stage, item generation and development of the scale were performed, based on the interview with panelists and respondents, as well as a review of the literature in this background relating to the protection motivation theory (PMT) for making an item pool and content domain. The principal dependent variable in the current analysis was the affecting factors on preventive behaviors toward COVID-19. Besides, the independent variables include five factors, organized into logical framework counting (a) Perceived severity, (b) Perceived vulnerability, (c) Perceived response cost, (d) Perceived response efficiency, and (e) Perceived selfefficacy. Then, the content and face validity assessment was done. In the next stage, the psychometric properties of the established scale were measured through a cross-sectional study on a sample of people. To complete this final stage, we done the exploratory factor analysis (EFA), confirmatory factor analysis (CFA), and analyzed item-scale correlation. At the end of the following part, the reliability of the AFPB-CO scale was measured, by internal consistency (through Cronbach's' alpha coefficient) and stability of the AFPB-CO scale, via test-retest.

\section{Phase 1: Item generation and scale development}

\section{Research design}

This stage of the study intended to develop a scale to measure the affecting factors of Iranian citizens in the preventive behaviors about COVID-19. We derived items employing the following three procedures: an exploration of the suitable number content domain, items' production, and the instrument design (11). The content domain discusses content related to the variables being assessed (12). The content domain generation emerged from a review of the related literature, as well as on interviews with the target group and professionals. The data collected from the interviews with the target group were applied to generate the instrument items $(13,14)$. The literature review reveals another method used to identify the content domain (13). In the other phase, item generation was carried out by the comment collected from the content domain. The following step of the study began the process of instrument structure, in which items were modified and suitably prepared. All accepted items were so arranged in an effective procedure (15). 
The item pool included 76-item at this point. It was tried to make the content of the items more comfortable and removed extra items through discussion. The chief researcher (MK) and other team members read items and deleted additional items. To the end, the first draft of the instrument was developed and contained with 51 -item. Then, content and face validity were assessed.

\section{Validity}

\section{Content validity.}

Qualitative and quantitative approaches were applied to assess content validity. In the qualitative phase, a panel of experts, including health promotion expert and epidemiologist, measured the content validity of the AFPB-CO scale. They evaluated phrasing, grammar, wording, item allocation, and scaling of the AFPB-CO scale. In the quantitative phase, the content validity index $(\mathrm{CVI})$ and the content validity ratio (CVR) of the AFPB-CO scale were evaluated. The CVI was assessed by requesting the experts to rate each item according to its simplicity, relevancy, and clarity (15) on a scale ranging from $1=$ not relevant, simple, and clear to $4=$ very relevant, simple, and clear. The CVI was measured as the proportion of items on the questionnaire that achieved a rating of three or four $(16,17)$. The CVR evaluated the essentiality of each item in the questionnaire. For measuring the CVR, the specialists rated each item as $1=$ essential, 2 = useful but not essential, or $3=$ not essential. Afterward, according to the Lawshe table, items with a CVR score of .99 or greater were determined to be acceptable and were maintained (15). In the quantitative measurement of the AFPB-CO scale, items with a CVR and a CVI more than .99 and .80, respectively, were preserved. 21-items were removed, resulting in a 39-item pool. The expert panel revised the AFPB-CO scale about wording, item allocation, and grammar as well.

\section{Face validity.}

Both qualitative and quantitative approaches were implemented for measuring the face validity. A group of laypeople $(n=10)$ was questioned to assess each item of the AFPB-CO scale and to indicate whether they experienced difficulty or confusion in answering the item. Afterward, to determine the percentage of laypeople who recognized items as important or quite important on a five-point Likert instrument, the impact score (importance $\times$ frequency) was measured. Items were determined to be suitable if they had an impact score of 1.5 or higher (which equals a mean rate of $50 \%$ and mean the importance of 3 out on 5) (18).

To sum up, three items had an impact score lower than 1.5. The range of impact score was from 1.6 to 5 . The first form of the AFPB-CO scale, including 36 items, was confirmed for the next stage of psychometric assessment. In other words, the group of laypeople showed that they experienced no problems reading and understanding the 36 -item of the AFPB-CO scale.

A pre-final version of the AFPB-CO scale: following the consideration of the above approaches, the prefinal version of the AFPB-CO scale, including 36-item, was produced for the subsequent stages (construct validity and reliability of the AFPB-CO scale). 


\section{Phase 2: Psychometric phase}

\section{i) The main study and data collection}

A cross-sectional study was conducted to measure the psychometric properties of the AFPB-CO scale in a more extensive background in 2020, in Mazandaran, Iran. Given the prevalence of pandemic COVID-19, we have faced restrictions on the traffic, and most of the public placed were closed in Iran, especially in Mazandaran province due to the high prevalence of COVID-19. Therefore, we tried to collect the study samples through cyberspace and social media. For this purpose, a AFPB-CO scale was designed online using Porsline software at https://survey.porsline.ir/\#/ (19).

Porsline is an online questionnaire creation software. It takes only a few drags and drops to create the questionnaire and form. After receiving the answer, we will receive instant statistical analysis and graphs. In the professional use of Porsline, we can direct them to relevant questions at any time, depending on the answer the respondents choose. Additionally, we can prevent one person from recording more than one response on a device, such as a mobile or laptop. The respondent will then be prompted with the message, "You have already answered this questionnaire; it is not possible to re-register the answer." We can also allow for a response but identify responses recorded more than once on a device as spam in the results table.

In the next step, the designed questionnaire link (https://survey.porsline.ir/s/56BXXtB) was shared in cyberspace and social networks (WhatsApp, Telegram, Instagram, Linkedln, Facebook, and Twitter). One of the items considered at the beginning of the questionnaire was the province and city of residence. All the people who lived in one of the cities of Mazandaran province were included in this study. The sample size was assessed based on Gable and Wolf's reference, which suggests that a sample of five to 10 people per item is needed to support a hypothetically fixed factor structure (20). Thus, the required sample size, i.e., 360 (36 items $\times$ ten persons) laypeople for each part of the analysis (EFA and CFA), were engaged using these approaches (overall 720 persons for construct validity).

\section{ii) Statistical analysis}

Several statistical methods were performed to measure the psychometric properties of the AFPB-CO scale. These were organized in the following instruction.

1. Construct validity: The construct validity was applied using exploratory factor analysis (EFA), confirmatory factor analysis (CFA), and item-scale correlation for the 36-items that remained.

Exploratory factor analysis (EFA)

An EFA was accomplished to find the main factors of the AFPB-CO scale. A sample of 360 lay people completed the online questionnaire of AFPB-CO. A principal component analysis (PCA) with varimax rotation was applied to extract the main factors. Bartlett's Test of Sphericity and Kaiser-Meyer-Olkin (KMO) were used to measure the suitability of the sample for the factor analysis. Additionally, the 
eigenvalues and scree plot were measured to specify how many dimensions to keep. One principle applicable for describing the number of dimensions to retain is the Kaiser's criterion, which is based on a rule of thumb. According to Kaiser's criterion, the criteria performed to extract the main dimensions had an eigenvalue of 1 or above, which categorized them as satisfactorily enough. Eigenvalues above one and scree plot were accompanied for finding the number of dimensions. Factor loadings $\geq .40$ were considered acceptable (21). There are no quick guidelines for defining the cut-off point, and the particular methods implemented were influenced mainly by our purposes for the current study. Most texts recommend a cut-off point of 0.4 . However, there are no definite approaches suggested to make such a purpose, and much depends on the scale being used. By contrast, Comrey and Lee (2013) recommended the performance of more stringent cut-off points, counting 0.32 (poor), 0.4-0.54 (fair), 0.55-0.62 (good), 0.63-0.70 (very good), or 0.71 and more (excellent) (22). In the current study, scores of.40 and more were considered as satisfactory for factor loadings.

\section{Confirmatory factor analysis (CFA)}

A CFA was performed to measure the coherence between the model and the data. A separate sample of 360 lay people completed the online questionnaire of AFPB-CO. The relative chi-square, comparative fit index (CFI), the goodness of fit index (GFI), root mean square error of approximation (RMSEA), standardized root mean square residual (SRMR), normed fit index (NFI), and non-normed fit index (NNFI) were measured to assess the model fit $(20,23)$. The $\mathrm{CFI}, \mathrm{GFI}, \mathrm{NNFI}$, and NFI values range between 0 and 1 , but values equal to or greater than .80 are generally considered satisfactory model fits. An RMSEA value between .08 and .10 indicates a mediocre fit, and a value lower than .08 indicates a good fit. Values smaller than .05 indicate a good fit for SRMR, but values between .05 and .08 and between .08 and .10 represent a close fit or a satisfactory fit, respectively (24).

\section{Item-scale correlation}

As a final point, item-scale correlations of AFPB-CO were evaluated to measure the degree to which each item was correlated to its dimension via the Spearman correlation coefficient. We estimated that, for each dimension of the AFPB-CO, the item scores of the dimension (e.g., perceived threat) would correlate more with the total score of the own dimension (e.g., perceived threat) instead of the total score of another dimension (e.g., motivational protection). Correlation values Zero - .20 are reflected poor; . 21 - .40, fair; $.41-.60$, good; $.61-.80$, very good; and beyond .81 , excellent (25).

\section{Reliability}

The internal consistency was evaluated, applying Cronbach's alpha coefficient for measuring the reliability of the AFPB-CO scale. The alpha values equal to or greater than .70 were considered acceptable (26). Additionally, the intraclass correlation coefficient (ICC) was assessed for evaluating the stability of the AFPB-CO scale. The AFPB-CO scale was read ministered by 25 citizens from Mazandaran province a week after the first completion of the AFPB-CO scale by the shared link of the AFPB-CO scale (https://survey.porsline.ir/s/56BXXtB) in cyberspace and social networks (WhatsApp, Telegram, and 
Instagram). ICC values equal to or higher than .40 are considered satisfactory (i's from .81 to 1.0 are excellent, from 0.61 to .80 are very good, from .41 to .60 are good, from .21 to .40 are fair, and from .0 to .20 are poor) (26). The analyses were performed using the statistical program SPSS for Windows version 24.0 and Amos 24.0.

\section{Scoring of AFPB-CO scale}

The final version of the AFPB-CO scale is revealed in Appendix 1. The items were rated on a five-point Likert-type scale, anchored at the extremes with one =completely agree to five=completely disagree for seven dimensions including perceived threat, responses costs, responses efficacy, rewards, efficacy, and motivation protection and with $1=$ never to $5=$ always (for the dimension of behavioral responses). Higher scores show more motivating to participation in the prevention of COVID-19. Eleven items were negatively worded (items $2,4,5,6,7,8,9,10,17,18$, and 19) and have to be reverse scored.

\section{Results}

\section{The study sample}

In this study, 720 lay people aged 18 years or older completed the AFPB-CO scale (360 participants for EFA and 360 participants for CFA). The mean age of the laypeople was 39.22 years (SD $=8.61)$. The 486 of the participants $(67.5 \%)$ were female, and $234(32.5 \%)$ of them were male. The $3.3 \%$ of $(n=24)$ participants were illiterate. Additionally, 20.6\% (148 participants) had only achieved a primary education level, 41.4\% (298 participants) had secondary education, and 34.72\% (250 participants) had higher educational level. About 26.9\% (194 participants) were unemployed, 26.1\% (188 participants) were householder, $2.2 \%$ ( $n=16)$ were retired, $16.4 \%$ (118 participants) had part-time jobs, and $28.3 \%$ (204 participants) worked full time. Regarding marital status, about $41.4 \%(n=298)$ were single, $46.4 \%(n=$ $334)$ were married, and $12.2 \%(n=88)$ were divorced. The most common source to gain information toward COVIS-19 was social media $(66.94 \%$ of participants $n=482)$. About $13.62 \%$ of participants $(n=98)$ reported that they obtained information from national media, and $19.44 \%$ of participants $(n=140)$ began using information from family and relatives. To buy essential items, 63.1\% (454 participants); go to work, $17.8 \%$ (128 participants); go to picnic and tourisms, $13.6 \%$ (98 participants); and other cases, $5.5 \%$ (40 participants) were the most common reason for going out of the house during the days of the Corona outbreak in Mazandaran Province by participants.

\section{Construct validity.}

\section{a. Exploratory factor analysis}

The measured KMO was .750, and Bartlett's test of sphericity was significant $(\chi 2=5276.468, p<.001)$, presenting the appropriateness of the sample for EFA. An eight-factor solution, with a 36-item scale, arisen, based on eigenvalues more than 1 and a loading level equal to 0.5 or more. Factor loadings of each item and the eight dimensions are offered in Table 2. All items were loaded on their dimensions. The 
eight dimensions of the AFPB-CO scale jointly accounted for $58.64 \%$ of the observed variance.

Additionally, the scree plot directed an eight-factor solution (Fig. 1). In total, an EFA was applied on the 36item of the AFPB-CO scale (cut-off point: .50).

As revealed in Table 1, eight dimensions were recognized: factor 1 (perceived threat), which includes 6 items (items 1-6); factor 2 (behavioral responses), which includes 5 items (item 32-36); factor 3 (perceived responses costs), which includes 6 items (items 11-16); factor 4 (fears), which includes 4 items (items 710); factor 5 (perceived responses efficacy), which includes 4 items (items 20-23); factor 6 (rewards), which includes 3 items (items 17-19); factor 7 (perceived self-efficacy), which includes 4 items (items 2831), and factor 8 (protection motivation), which includes 4 items (items 24-27). Please refer to the Appendix to read the items of the AFPB-CO in full.

\section{b. Confirmatory factor analysis}

We conducted a CFA on the 36-item AFPB-CO scale to assess the fitness of the model obtained from the EFA. Based on Figure 2, the best model fit was obtained. Covariance matrixes were performed, and fit indices were measured. All fit indices were found to be appropriate. The relative chi-square ( $\chi 2 / \mathrm{df})$ was equal to $2.692(p<.001)$. The RMSEA of the model was $.069(90 \% \mathrm{Cl}=.06-.07)$, and the SRMR was .06 . All comparative indices in the structural model, containing $\mathrm{GFI}, \mathrm{AGFI}, \mathrm{CFI}$, and $\mathrm{NFI}$, were more than .90 $(.89, .91, .83$, and .80 , respectively). Even though the model fit was acceptable and good, modification indices for the regression weights were tested to find covariance among the eight factors. There was no achievement of any significant enhancement on fit indices; thus, no variations were performed, and the model was accepted in its existing form. Figure 2 demonstrates the model.

\section{Item-scale correlation}

Table 2 shows the item-scale correlation for the AFPB-CO scale. As can be observed, all coefficients are higher than .30, and most of them are higher than 0.50. Perceived threat and efficacy had the highest and the lowest item-scale correlation, respectively.

\section{Reliability}

The Cronbach's alpha was tested separately for the AFPB-CO scale and each dimension of the AFPB-CO scale in order to assess the internal consistency of the AFPB-CO scale. The Cronbach's alpha coefficient for the AFPB-CO scale ranged from .62 to .84 for its dimensions, which is more than the acceptable threshold. Additionally, the test-retest analysis was applied to assess the stability of the AFPB-CO scale. The results showed a satisfactory threshold. Intra-class correlation (ICC) ranged from .73 to .87 for the dimensions of the AFPB-CO scale, giving support for the scale's stability. The results are available in Table 3.

\section{Discussion}


In the current study, we defined the development and psychometric properties of a new scale, called the AFPB-CO, for measuring factors that affect persons' participation in prevention behaviors toward COVID19. This is the first project to extend a measure for assessing the factors related to participate in preventive behaviors of COVID-19 in the world, especially in the Iranian population. The content of the AFPB-CO scale items was first developed based on the interviews with the experts/laypeople and the review of the literature to ensure that this instrument covered all theoretical concepts linked to the protection motivation theory (PMT). One of the approaches to scale design, especially in the area of preventive behaviors, is the use of motivational theories like the protection motivation theory (PMT). Overall, the PMT makes available a valued theoretical structure for dealing with the difficulties of preventive behaviors. PMT can be performed to describe behavior associated with threat and coping related to health dangers and behavioral intention. Concepts such as perceived severity, perceived vulnerability, perceived response cost, perceived response efficiency, and perceived self-efficacy are the key constructs of the PMT. The assessment of theoretical constructions is one of the most difficult and necessary components in the study of theory-based health education and promotion (10). By knowing cognitive-behavioral principles, elements influencing the encouragement to do determined behaviors are recognized and impacted in the intervention researches. In the current project, the PMT components were measured by the complex technique. These components are one of the most common reasons to do the prevention of disease people might consider themselves at risk of possible comorbidities. The PMT may be applied to advise effective communications to reform existing postoperative recommendations (10). Thus, according to the reports mentioned above, PMT can be a suitable theory to design interventional programs.

Overall, the findings indicated satisfactory psychometric properties for the AFPB-CO scale. The CVI and the CVR indicated that the content validity of the AFPB-CO scale was sensible. The results of the analysis due to the KMO index show adequate sample size and satisfactory factor analysis. Furthermore, the results of the EFA and CFA showed a good structure for the AFPB-CO scale. After EFA, an eight-domain scale including perceived threat, behavioral responses, perceived response costs, fears, perceived responses efficacy, rewards, perceived self-efficacy, and protection motivation extracted accounted for 58.64 of the variance and the maximum stated variations were linked to the perceived threat as a first domain. Threat references to the extent to which persons perceive they are vulnerable to the health danger and their opinion of the sternness of the health danger (10). This shows that the AFPB-CO scale is useful for introducing various aspects of the health concerns influenced by participation in preventive behaviors.

The CFA was also used to specify whether there was coherence between the information and the theoretical structure. The CFA revealed good fit indices for the existing model, and the convergent validity of the eight subscales of the AFPB-CO scale was acceptable. As such, the final AFPB-CO scale contained 36 items, with seven items indicating outcome evaluations, six items representing a perceived threat, five items representing behavioral responses, six items representing responses costs, four items expressing fears, four items describing responses efficacy, three items representing rewards, four items representing efficacy, and three items representing protection motivation of preventive behaviors toward COVID-19. 
Additionally, the internal consistency of the AFPB-CO scale, as evaluated by the Cronbach's alpha, displayed suitable reliability for two dimensions. Moreover, after testing 30 laypeople over a week period, our results clearly showed that the AFPB-CO scale has appropriate stability in the short term; however, it has yet to be perceived whether the AFPB-CO scale is stable for a long time.

Overall, the results confirmed that this newly developed AFPB-CO scale is a suitable and valid scale that can be used for measuring participating in preventive behavior toward COVID-19 among lay people who speak Farsi. Developing theory-based scales can be used as a significant necessity for the calculation of any educational program. Therefore, we consider the results from the present study to be valuable for clients who are part of a COVID-19 preventive plan. On the other hand, it may be principally useful to health care experts to identify and design processes that are beneficial and targeted to particular conditions. The inclusion of eight domains in the AFPB-CO scale further lets specialists identify and decide how components in which a person can be improved.

\section{Limitations}

Although the current study has several strengths, it has some limitations. First, the present study was done among a sample of people from Mazandaran province (north of Iran) to express affecting factors on their participation in performing preventive behaviors about COVID-19. Due to this, we cannot be definite that the conclusions perform to laypeople in another geographic background. Consequently, further researches may be needed to support the applicability of the theory-based scale of AFPB-CO as a fully confirmed applied and useful measure in the Iranian context. Second, the Cronbach's alpha coefficients of some factors were not satisfactory. Future studies are needed to overcome these problems. The third one is related to the truthfulness of responses and the self-reported nature of the scale. The generalizability and sample size made up another limitation of the present study. The current sample was limited to a group of 720 (both EFA and CFA) laypeople, and it is uncertain whether we would attain the same results if a large sample of both people were employed. Finally, the present study included only persons who were referred to the online link of scale and who completed the questionnaire online and virtual. There may be many populations who do not have a smartphone or Internet access to complete a questionnaire. Furthermore, it can be interesting for future studies with a larger sample to test whether the psychometric properties of the instrument hold with an alternative measure of reliability and validity (e.g., test-retest validity).

In summary, one of the objectives of the Century is increasing participation of people in preventive behaviors toward COVID-19. To do so, we developed the AFPB-CO scale, which was revealed to have acceptable psychometric properties.

\section{Conclusion}

Generally, the AFPB-CO scale indicated good construct validity, and the majority of domains showed high internal consistency reliability; thus, the results of the current study offer that a theory-based AFPB-CO 
scale is a valid and reliable scale for assessing affecting factors on participation of people in preventive behaviors about COVID-19. Furthermore, the Iranian version of the AFPB-CO scale may be helpful for health care workers in finding and planning health strategies that are useful and targeted to particular statuses. Additionally, more studies are proposed to identify the strengths and weaknesses of the AFPB$\mathrm{CO}$ scale when it is performed in other contexts.

\section{Abbreviations}

BR: Behavioral responses; AFPB-CO: Affecting factors on preventive behaviors of COVID-19; PMT: Protection motivation theory; PT: Perceived threat; PRC: Perceived response cost; PRE: Perceived response efficiency; PSE: perceived self-efficacy; F: Fears; R: rewards; PM: Protection motivation; CVI: Content validity index; CVR: Content validity ratio; EFA: Exploratory factor analysis; CFA: Confirmatory factor analysis; GFI: Goodness of Fit Index, CFI: Comparative Fit Index; RMSEA: Root Mean Square Error of Approximation; NNFI: Non-Normed Fit Index; NFI: Normed Fit Index; SRMR: Standardized Root Mean Square Residual; ICC: Intraclass correlation coefficient.

\section{Declarations}

\section{Ethics approval and participation consent}

The study procedure was approved by the Medical Ethics Committee of Mazandaran University of Medical Sciences [IR.MAZUMS.REC.1398.7263]. This study involved only persons who gave their informed consent. For this, all participants completed an informed consent form before completing the scale. The AFPB-CO scale was treated as private and nameless; there was no personal information that could link the answers with any of the participants in the present study. No records of consent by name were kept to protect the rights and interests of all participants.

\section{Consent for publication}

Not applicable.

\section{Availability of data and materials}

The datasets produced and analyzed throughout the current study are not publicly available in order to keep the participants' privacy but are available from the corresponding author on reasonable request.

\section{Competing interests}

The authors declare that they have no competing interests.

\section{Funding}

No external funding sources were provided for this manuscript. 


\section{Authors' contribution}

MK designed the project, did the statistical analysis, and wrote the first draft of the manuscript. AN and TP participated in developing the project and collected the data. LJ contributed to the item generation. MD contributed to the collected data. KP contributed to the statistics. MK and KP critically revised the final article. The author read the final paper and agreed to be accountable for all aspects of the work in ensuring that questions related to the accuracy or integrity of any part of the work are appropriately investigated and resolved.

\section{Acknowledgments}

We would like to thank all Citizens of Mazandaran for their participation in the current study, as well as the panel experts, without whose support the present study could not have been performed. Likewise, the authors are thankful for the support received from the Deputy of Research and Technology, Mazandaran University of Medical Sciences (MAZUMS).

\section{Authors' Information}

Maryam Khazaee-Pool, Department of Public Health, School of Health, Mazandaran University of Medical Sciences, Sari, Iran; Health Sciences Research Center, Addiction Research Institutes, Mazandaran University of Medical Sciences, Sari, Iran; Seyed Abolhassan Naghibi, Department of Public Health, School of Health, Mazandaran University of Medical Sciences, Sari; Tahereh Pashai, Department of Public Health, School of Health, Kurdistan University of Medical Sciences, Sanandaj, Iran; Leila Jahangiri, Health Education and Health Promotion Department, School of Public Health, Tabriz University of Medical Sciences, Tabriz, Iran; Mahbobeh Daneshnia, Department of Public Health, School of Public Health, Mazandaran University of Medical Sciences, Sari, Iran; Koen Ponnet, Department of Communication Studies, imec-mict, Ghent University, Ghent, Belgium.

\section{References}

1. Hui DS, Azhar I, Madani E, Ntoumi TA, Kock F, Dar R. O, et al. The continuing 2019-nCoV epidemic threat of novel coronaviruses to global health-The latest 2019 novel coronavirus outbreak in Wuhan, China. International Journal of Infectious Diseases. 2020;91:264-6.

2. Kassema JJ. COVID-19 Outbreak: Is It a Health Crisis or Economic Crisis or Both? Case of African Counties. Case of African Counties (23 Mar, 2020). 2020.

3. Wu Z, McGoogan JM. Characteristics of and important lessons from the coronavirus disease 2019 (COVID-19) outbreak in China: summary of a report of 72314 cases from the Chinese Center for Disease Control and Prevention. Jama. 2020.

4. Tomasi D. Coronavirus disease (COVID-19). A socioepidemiological analysis Bennington. VT: Vermont Academy of Arts and Sciences LV; 2020.

5. Hu Z, Yang Z, Li Q, Huang Y. Nomenclature: coronavirus and the 2019 novel coronavirus. 2020. 
6. Adhikari SP, Meng S, Wu Y-J, Mao Y-P, Ye R-X, Wang Q-Z, et al. Epidemiology, causes, clinical manifestation and diagnosis, prevention and control of coronavirus disease (COVID-19) during the early outbreak period: a scoping review. Infectious Diseases of Poverty. 2020;9(1):1-12.

7. Tavakoli A, Vahdat K, Keshavarz M. Novel Coronavirus Disease 2019 (COVID-19): An Emerging Infectious Disease in the 21st Century. ISMJ. 2020;22(6):432-50.

8. Jin Y-H, Cai L, Cheng Z-S, Cheng H, Deng T, Fan Y-P, et al. A rapid advice guideline for the diagnosis and treatment of 2019 novel coronavirus (2019-nCoV) infected pneumonia (standard version). Military Medical Research. 2020;7(1):4.

9. Dhama K, Sharun K, Tiwari R, Sircar S, Bhat S, Malik YS, et al. Coronavirus disease 2019-COVID-19. 2020.

10. Rogers RW. Cognitive and psychological processes in fear appeals and attitude change: A revised theory of protection motivation. Social psychophysiology: A sourcebook. 1983:153-76.

11. Nunnally JC. Psychometric theory 3E: Tata McGraw-Hill Education; 1994.

12. Beck CT, Gable RK. Ensuring content validity: An illustration of the process. J Nurs Meas. 2001;9(2):201-15.

13. Newman I, Lim J, Pineda F. Content validity using a mixed methods approach: Its application and development through the use of a table of specifications methodology. Journal of Mixed Methods Research. 2013;7(3):243-60.

14. Tilden VP, Nelson CA, May BA. Use of qualitative methods to enhance content validity. Nursing Research. 1990.

15. Lynn MR. Determination and quantification of content validity. Nursing research. 1986.

16. Waltz CF, Bausell BR. Nursing research: design statistics and computer analysis: Davis FA; 1981.

17. Lawshe CH. A quantitative approach to content validity 1. Personnel psychology. 1975;28(4):56375.

18. Lacasse Y, Godbout C, Series F. Health-related quality of life in obstructive sleep apnoea. Eur Respir J. 2002;19(3):499-503.

19. https://survey.porsline.ir/\#/. 2020.

20. Gable RK, Wolf MB. A Review of the steps for developing an affective instrument. Instrument Development in the Affective Domain: Springer; 1993. p. 237 - 66.

21. Norris M, Lecavalier L. Evaluating the use of exploratory factor analysis in developmental disability psychological research. J Autism Dev Disord. 2010;40(1):8-20.

22. Comrey AL, Lee HB. The first course in factor analysis: Psychology press; 2013.

23. Harrington D. Confirmatory factor analysis: Oxford university press; 2009.

24. Kline RB. Principles and practice of structural equation modeling: Guilford publications; 2015.

25. Strube MJ. Reliability and generalizability theory. 2000.

26. Cronbach LJ. Coefficient alpha and the internal structure of tests. psychometrika. 1951;16(3):297334. 
Tables

Table 1: Exploratory factory analysis of the AFPB-CO $(n=360)$

\begin{tabular}{|c|c|c|c|c|c|c|c|c|}
\hline mber of Items & Factor 1 & Factor 2 & Factor 3 & Factor 4 & Factor 5 & Factor 6 & Factor 7 & Factor 8 \\
\hline 1 & .918 & .025 & .024 & -.045 & -.006 & .002 & .025 & -.067 \\
\hline 6 & .884 & .026 & .022 & -.047 & .000 & .006 & .004 & -.054 \\
\hline 3 & .781 & .023 & .094 & -.079 & .008 & -.010 & -.072 & .014 \\
\hline 5 & .774 & .048 & .082 & -.056 & .005 & -.057 & .013 & .081 \\
\hline 4 & .770 & .009 & .116 & .014 & -.027 & -.095 & .036 & .157 \\
\hline 2 & .712 & .044 & .033 & -.081 & -.052 & .016 & .050 & .037 \\
\hline .36 & .009 & .839 & -.077 & .035 & -.052 & -.068 & -.021 & .089 \\
\hline .32 & -.008 & .805 & -.034 & .013 & -.021 & -.040 & .017 & .097 \\
\hline .33 & -.060 & -.794 & .073 & -.027 & -.034 & -.023 & .024 & .079 \\
\hline .35 & .035 & .751 & -.039 & -.020 & -.049 & -.051 & -.004 & -.097 \\
\hline .34 & -.065 & -.728 & .064 & -.051 & .036 & .044 & -.020 & -.035 \\
\hline C.13 & .061 & -.013 & .745 & -.086 & -.060 & .019 & .042 & -.094 \\
\hline C.16 & .057 & -.086 & .744 & -.059 & -.094 & .031 & -.036 & .090 \\
\hline C.11 & -.029 & -.055 & -.714 & .016 & -.100 & -.044 & .002 & -.012 \\
\hline C.12 & .102 & .003 & .684 & -.003 & -.021 & -.003 & -.005 & -.054 \\
\hline C.14 & .094 & -.128 & .676 & -.131 & -.026 & .088 & -.057 & -.062 \\
\hline C.15 & .001 & .134 & -.626 & -.001 & .035 & .123 & -.020 & -.015 \\
\hline 9 & -.038 & .059 & -.073 & .811 & .017 & -.046 & -.014 & .011 \\
\hline 8 & .047 & .031 & .085 & -.808 & -.037 & -.041 & .030 & .006 \\
\hline 7 & -.110 & -.053 & -.114 & .797 & .039 & .105 & -.065 & -.012 \\
\hline 10 & -.078 & .135 & .004 & .785 & .080 & -.033 & -.008 & .028 \\
\hline E.20 & -.006 & .074 & .024 & .094 & .789 & -.003 & .016 & .049 \\
\hline E.21 & .065 & .055 & .037 & -.014 & -.786 & .000 & .046 & -.035 \\
\hline E.22 & -.075 & -.084 & -.091 & .012 & .779 & .069 & -.046 & .049 \\
\hline E.23 & .081 & -.053 & -.018 & .042 & .770 & -.030 & .092 & -.050 \\
\hline 17 & -.034 & -.041 & .041 & -.034 & .039 & .924 & .139 & .043 \\
\hline 19 & -.039 & -.073 & .046 & -.008 & .032 & .923 & .098 & .041 \\
\hline 18 & .066 & .088 & .057 & -.122 & .056 & -.438 & .120 & .261 \\
\hline E.30 & -.010 & -.021 & -.001 & -.017 & -.059 & -.225 & -.780 & -.061 \\
\hline Е.29 & .003 & -.011 & -.023 & .019 & -.029 & -.013 & .777 & .030 \\
\hline E.31 & .037 & -.043 & .014 & -.068 & .046 & .134 & .663 & -.029 \\
\hline E.28 & .007 & -.033 & .027 & .076 & .062 & .267 & -.559 & .044 \\
\hline .25 & -.091 & -.068 & -.038 & -.090 & .097 & -.012 & .008 & -.639 \\
\hline .26 & -.055 & -.070 & -.036 & .104 & .091 & -.046 & -.009 & .530 \\
\hline .24 & -.053 & -.040 & -.051 & .190 & -.068 & -.191 & -.057 & -.502 \\
\hline .27 & .044 & -.002 & -.115 & -.032 & .003 & -.129 & -.025 & .502 \\
\hline
\end{tabular}

Note. Figures in bold relate to factor loadings equal to or greater than .50.

Table 2: Item-scale correlation matrix for the eight AFPB-CO dimensions $(n=360)$ 


\begin{tabular}{|c|c|c|c|c|c|c|c|c|}
\hline mber of Items & $P T$ & $\mathrm{Fe}$ & $P R C$ & $R e$ & $P R E$ & $P M$ & PSE & $B R$ \\
\hline 1 & $.870^{* *}$ & -.131 & .121 & -.019 & .014 & -.071 & .083 & -.047 \\
\hline 2 & $.765^{* *}$ & -.186 & .138 & -.014 & -.064 & -.052 & .043 & -.009 \\
\hline 3 & $.779^{* * *}$ & -.135 & .152 & -.002 & .002 & -.071 & .003 & -.021 \\
\hline 4 & $.812^{* *}$ & -.118 & .119 & -.012 & -.011 & -.076 & .036 & .063 \\
\hline 5 & $.806^{* *}$ & -.074 & .075 & -.025 & .015 & -.072 & -.010 & .014 \\
\hline 6 & $.851^{* *}$ & -.134 & .108 & .003 & .034 & -.073 & .049 & -.061 \\
\hline 7 & -.188 & $.759^{* *}$ & -.143 & -.021 & .048 & .059 & -.028 & -.092 \\
\hline 8 & .111 & $.342^{* *}$ & .165 & .043 & -.050 & -.040 & .024 & .040 \\
\hline 9 & -.070 & $.782^{* *}$ & -.109 & -.066 & .090 & .092 & .019 & .013 \\
\hline 10 & -.093 & $.769^{* *}$ & -.154 & -.059 & .080 & .036 & -.008 & .051 \\
\hline \multicolumn{9}{|l|}{$C$} \\
\hline C.11 & -.062 & .060 & $.584^{* *}$ & -.139 & -.031 & .109 & -.016 & -.005 \\
\hline C.12 & $.122^{*}$ & -.025 & $.665^{* *}$ & .043 & -.040 & -.028 & .039 & -.006 \\
\hline C.13 & $.128^{*}$ & -.125 & $.634^{* *}$ & .063 & -.045 & -.047 & .036 & -.047 \\
\hline C.14 & .086 & -.167 & $.633^{* *}$ & .081 & -.068 & -.131 & .014 & -.072 \\
\hline C.15 & -.026 & .028 & $.670^{* *}$ & -.006 & .064 & .022 & -.023 & .009 \\
\hline C.16 & .103 & $-.117^{*}$ & $.679^{* *}$ & .089 & -.016 & -.079 & .000 & -.063 \\
\hline 17 & -.084 & -.033 & .063 & $.847^{* *}$ & .026 & -.090 & .043 & -.089 \\
\hline 18 & .131 & -.035 & -.043 & $.550^{* *}$ & .011 & .027 & -.110 & .057 \\
\hline 19 & -.111 & -.020 & .057 & $.848^{* *}$ & .005 & -.086 & .037 & -.070 \\
\hline \multicolumn{9}{|l|}{$\mathrm{E}$} \\
\hline E.20 & -.022 & .100 & -.053 & .009 & $.675^{* *}$ & .031 & -.031 & -.053 \\
\hline E.21 & .076 & -.040 & .111 & -.040 & $-.571^{* *}$ & -.038 & .027 & .042 \\
\hline E.22 & -.091 & .037 & -.109 & .048 & $.674^{* *}$ & .036 & .017 & -.058 \\
\hline E.23 & .017 & .014 & -.082 & .022 & $.705^{* *}$ & .048 & .011 & -.079 \\
\hline \multicolumn{9}{|l|}{$\bar{r}$} \\
\hline .24 & -.074 & .091 & -.018 & -.171 & .016 & $.589^{* *}$ & .003 & -.004 \\
\hline .25 & $-.136^{*}$ & -.013 & -.015 & .039 & -.066 & $.455^{* *}$ & -.052 & -.044 \\
\hline .26 & -.028 & .086 & -.045 & .003 & .074 & $.460^{* *}$ & .043 & -.003 \\
\hline .27 & .082 & -.031 & -.040 & .004 & .074 & $.346^{* *}$ & -.032 & .074 \\
\hline \multicolumn{9}{|l|}{$E$} \\
\hline E.28 & .002 & .030 & .053 & .022 & -.037 & -.042 & $.510^{* *}$ & -.084 \\
\hline E.29 & .016 & -.008 & .021 & .056 & .022 & .012 & $.346^{* *}$ & .048 \\
\hline E.30 & -.006 & .009 & .009 & $-.279^{* *}$ & -.042 & .010 & $.313^{*}$ & .042 \\
\hline E.31 & .082 & -.095 & .002 & .120 & .096 & -.007 & $.424^{* *}$ & -.002 \\
\hline .32 & .066 & .114 & -.085 & -.067 & -.044 & -.049 & -.059 & $.577^{* *}$ \\
\hline .33 & -.118 & -.073 & .063 & .032 & .058 & .047 & -.005 & $.317^{* *}$ \\
\hline .34 & -.130 & -.066 & .046 & .037 & .040 & .011 & .037 & $.334^{*}$ \\
\hline .35 & .097 & .026 & -.048 & -.056 & -.111 & .042 & .058 & $.637^{* *}$ \\
\hline
\end{tabular}


Table 3: Measures of the internal consistency and intraclass coefficient

\begin{tabular}{l|l|l|c|c|}
\hline 7actor & \multicolumn{1}{|c|}{ The name of factor } & Number of items & Cronbach's alpha & ICC \\
\hline$\vdots$ & Perceived threat & 6 items (1-6) & .84 & .85 \\
\hline & Behavioral responses & 5 items (32-36) & .82 & .87 \\
\hline & Perceived Responses costs & 6 Items (11-16) & .79 & .84 \\
\hline$;$ & Fears & 4 items (7-10) & .80 & .79 \\
\hline$;$ & Perceived Responses efficacy & 4 Items (20-23) & .77 & .73 \\
\hline :otal & Rewards & 3 Items (17-19) & .62 & .81 \\
\hline & Perceived Self-Efficacy & 4 items (28-31) & .71 & .78 \\
\hline & Protection motivation & 4 items (24-27) & .74 & .84 \\
\hline
\end{tabular}

\section{Appendix}

Perceived threat

Q1. Like all people in the world, I may be infected with Corona.

Q2. Only the elderly and those with chronic illnesses are affected by Corona.

Q3. I may be infected with Corona as a result of contact with outdoor surfaces (shopping center - gas station - workplace).

Q4. Staying healthy or having a corona depends on my luck and fate.

Q5. The result of Corona disease can be deadly because there is no definitive cure for it.

Q6. The result of Corona disease is severe damage to my lungs that disrupts my life

\section{Fears}

Q7. I'm more worried about my family having Corona. ) Most of all, I am concerned about my family being infected with Corona.)

Q8. I get anxious if I come in contact with different surfaces (e.g., elevator door, home door - shopping center - gas station - workplace).

Q9. I am scared of even going out of the house for essential work.

Q10. If I have Corona, I have to spend long days in the hospital with anxiety. 
Q11. Continuous washing of hands at least 20 seconds outside the home is time-consuming and difficult.

Q12. If I do not shake hands or I have a distance with them when communicating with others, they may be angry with me.

Q13. When I wear gloves, my hands sweat.

Q14. It is hard to breathe with a mask.

Q15. Washing regularly my hands with soap makes my skin more sensitive.

Q16. If I stay at home and be a quarantine, I get depressed and bored.

Rewards

Q17. In these days of Corona outbreaks, traveling or leaving home makes me happy.

Q18. When I shake hands with others or kiss them, I feel more intimate.

Q19. Eating cold food and bread is more enjoyable than cooked food and hot bread.

Perceived Responses efficacy

Q20. Washing my hands with soap and water for at least 20 seconds is an excellent way to prevent me from Corona disease.

Q21. Wearing masks and gloves outdoors and in crowded places prevent me from transmitting Corona.

Q22. Maintaining my physical and social distance with others (2 meters) helps break the Corona transmission chain.

Q23. Disinfecting the surfaces of my home and workplace protects me against Corona.

Protection motivation

Q24. I am going to disinfect all the nuts and the stuff I buy outside the house.

Q25. I want to keep myself and others healthy by being 2 meters away.

Q26. As long as there is a risk of Corona transfer, I plan to keep in touch with family and friends online.

Q27. I'm going to warm the food and bread before eating well.

Perceived Self-Efficacy

Q28. I can easily wash my hands before contacting my face and after contact with surfaces outdoors. 
Q29. If I have had a fever, shortness of breath, or cough, I can go to the hospital immediately or talk to a doctor.

Q30. Without any problems, I can use clean masks and gloves when I get out of the house and into crowded places.

Q31. I can stay home for as long as I have a chance of getting a corona, even if I'm upst and frustrated.

Behavioral Responses

Q32. When exposed to different surfaces, I wash or disinfect my hands with soap and water for at least 20 seconds.

Q33. To cut the Corona transmission chain, I put the dirty gloves and masks after use in the trash bin.

Q34. I regularly disinfect different levels of contact (home - car - work - pedestrian card, mobile phone, etc.).

Q35. When touching contaminated surfaces, I take care not to touch my eyes, mouth, and nose with my hands.

Q36. I change clothes outside and put them outside before I get inside the home.

Perceived threat

Q1. Like all people in the world, I may be infected with Corona.

Q2. Only the elderly and those with chronic illnesses are affected by Corona.

Q3. I may be infected with Corona as a result of contact with outdoor surfaces (shopping center - gas station - workplace).

Q4. Staying healthy or having a corona depends on my luck and fate.

Q5. The result of Corona disease can be deadly because there is no definitive cure for it.

Q6. The result of Corona disease is severe damage to my lungs that disrupts my life

Fears

Q7. I'm more worried about my family having Corona. ) Most of all, I am concerned about my family being infected with Corona.)

Q8. I get anxious if I come in contact with different surfaces (e.g., elevator door, home door - shopping center - gas station - workplace). 
Q9. I am scared of even going out of the house for essential work.

Q10. If I have Corona, I have to spend long days in the hospital with anxiety.

Perceived Responses costs

Q11. Continuous washing of hands at least 20 seconds outside the home is time-consuming and difficult.

Q12. If I do not shake hands or I have a distance with them when communicating with others, they may be angry with me.

Q13. When I wear gloves, my hands sweat.

Q14. It is hard to breathe with a mask.

Q15. Washing regularly my hands with soap makes my skin more sensitive.

Q16. If I stay at home and be a quarantine, I get depressed and bored.

Rewards

Q17. In these days of Corona outbreaks, traveling or leaving home makes me happy.

Q18. When I shake hands with others or kiss them, I feel more intimate.

Q19. Eating cold food and bread is more enjoyable than cooked food and hot bread.

Perceived Responses efficacy

Q20. Washing my hands with soap and water for at least 20 seconds is an excellent way to prevent me from Corona disease.

Q21. Wearing masks and gloves outdoors and in crowded places prevent me from transmitting Corona.

Q22. Maintaining my physical and social distance with others (2 meters) helps break the Corona transmission chain.

Q23. Disinfecting the surfaces of my home and workplace protects me against Corona.

Protection motivation

Q24. I am going to disinfect all the nuts and the stuff I buy outside the house.

Q25. I want to keep myself and others healthy by being 2 meters away.

Q26. As long as there is a risk of Corona transfer, I plan to keep in touch with family and friends online. 
Q27. I'm going to warm the food and bread before eating well.

Perceived Self-Efficacy

Q28. I can easily wash my hands before contacting my face and after contact with surfaces outdoors.

Q29. If I have had a fever, shortness of breath, or cough, I can go to the hospital immediately or talk to a doctor.

Q30. Without any problems, I can use clean masks and gloves when I get out of the house and into crowded places.

Q31. I can stay home for as long as I have a chance of getting a corona, even if I'm upst and frustrated.

Behavioral Responses

Q32. When exposed to different surfaces, I wash or disinfect my hands with soap and water for at least 20 seconds.

Q33. To cut the Corona transmission chain, I put the dirty gloves and masks after use in the trash bin.

Q34. I regularly disinfect different levels of contact (home - car - work - pedestrian card, mobile phone, etc.).

Q35. When touching contaminated surfaces, I take care not to touch my eyes, mouth, and nose with my hands.

Q36. I change clothes outside and put them outside before I get inside the home.

\section{Figures}




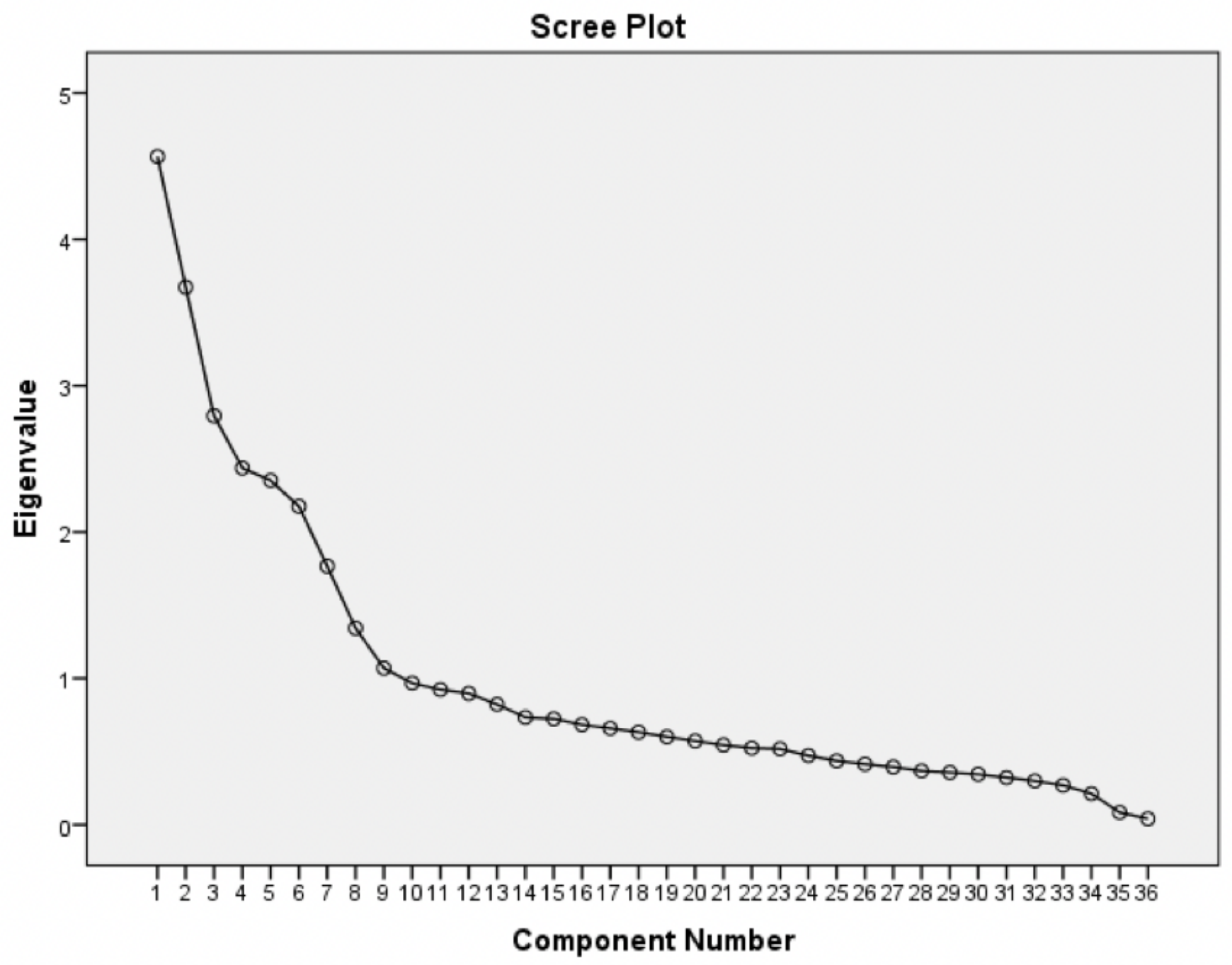

Figure 1

Scree plot for determining factors of the designed instrument. 


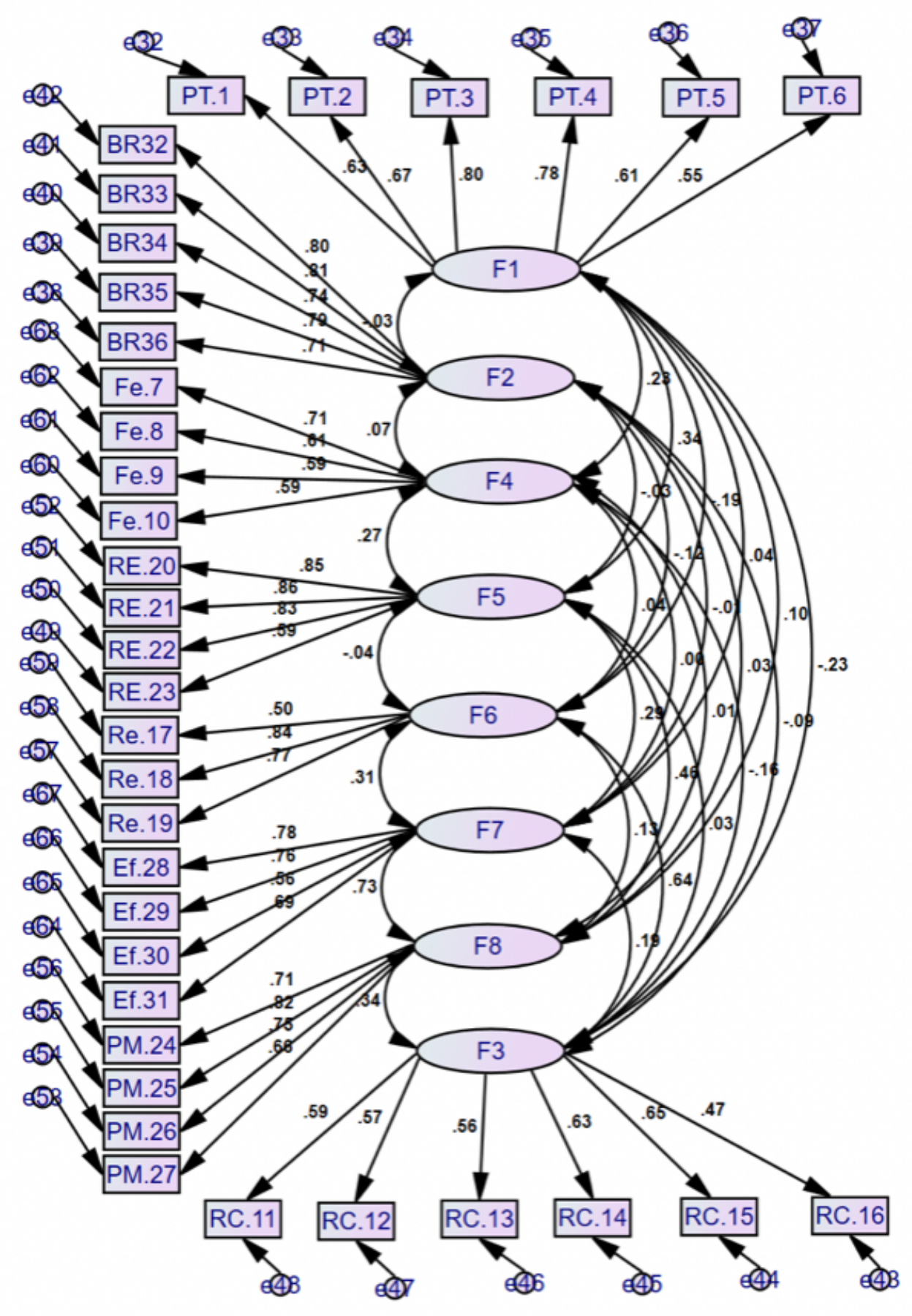

Figure 2

An eight-factor model for the scale gained from the confirmatory factor analysis $(n=360)$. 